\title{
ESTUDIO DE LOS MICRODESECHOS LÍTICOS DEL AURIÑACIENSE DE TRANSICIÓN (NIVEL 18B) DE LA CUEVA DE EL CASTILLO (PUENTE VIESGO, CANTABRIA)
}

\author{
ANALYSIS OF LITHIC MICRODEBRIS FROM THE TRANSITIONAL AURIGNACIAN \\ (LEVEL 18B) AT CASTILLO'S CAVE (PUENTE VIESGO, CANTABRIA)
}

\author{
ALBERTO MINGO (*) \\ JESÚS BARBA $(* *)$ \\ ROBERTO PERETTI (***)
}

\section{RESUMEN}

A través del análisis de los microdesechos líticos se puede identificar aspectos relacionados con el aprovechamiento y uso de materias primas; constatar el desarrollo de actividades enfocadas a la elaboración, modificación y mantenimiento opcional de los útiles; inferir las diferentes técnicas de talla empleadas; observar, a través de mapas de densidad, la distribución espacial de los microdesechos, y entender mejor la dinámica de formación de los registros arqueológicos. En este trabajo se han estudiado los microdesechos líticos del nivel 18b (Auriñaciense de Transición) de la cueva de El Castillo. En él se presentan la metodología empleada y los resultados obtenidos.

\section{ABSTRACT \\ Through the analysis of microdebitage we are able to identify features concerning the exploitation and use of raw materials; the verification of manufacture, use, modifica- tion and maintenance activities of tools, to infer the diffe- rent knapping techniques employed; to observe, through density maps, the spatial distributions of microdebris, and better understand the formation of archaeological records. In this paper, we have studied the lithic microdebitage of}

(*) Departamento de Prehistoria e Historia Antigua. Becario Predoctoral-UNED. Paseo Senda del Rey, s/n. 28040 Madrid. España. Correo electrónico: amingo@bec.uned.es

(**) Departamento de Prehistoria e Historia Antigua. Becario Predoctoral-MEC. Paseo Senda del Rey, s/n. 28040 Madrid. España. Correo electrónico: jbarba@bec.uned.es

(***) Departamento de Arqueología INCUAPA. UNC. Del Valle 5737. B7400 JWI Olavarría. Argentina. Correo electrónico: rpereti@soc.unicen.edu.ar

Recibido: 8-II-03; aceptado:27-VII-03. level $18 b$ (Aurignacian of Transition) from Cueva de El Castillo and present the methodology applied and the results obtained.

Palabras clave: Microdesechos líticos. Auriñaciense de Transición. Cantabria. Cadena operativa. Materias primas. Aspectos tecnológicos. Procesos postdeposicionales. Distribuciones espaciales.

Key words: Microdebitage. Transitional Aurignacian . Cantabria. Lithic production system. Raw materials. Technological features. Postdepositional processes. Spatial distributions.

\section{INTRODUCCIÓN}

Este estudio que aquí se presenta es la continuación del que llevaron a cabo Peretti y Mingo (2000) sobre los microdesechos líticos provenientes de los niveles 18c, 18c Base y 19 Superior del yacimiento de la cueva de El Castillo. Ambos trabajos se enmarcan dentro de los proyectos de investigación " $\mathrm{El}$ final de los Neandertales: La transición cultural en Cantabria y su contexto europeo. Una visión multidisciplinar" (BHA 2000-00200) de la OCYT y "Cambios sociales y condiciones paleoambientales en el Pleistoceno Superior de Cantabria: Monte Castillo y su contexto" de la UNED, dirigidos por la Dra. Victoria Cabrera Valdés. Estas investigaciones, también apoyadas por la Consejería de Cultura del Gobierno de Cantabria tienen por objeto la obtención de información relevante que permita la contrastación o identificación de modelos de desa- 
rrollo cultural en la transición del Paleolítico Medio al Superior en el área Cantábrica.

Este trabajo aborda específicamente el análisis de los microdesechos líticos provenientes del nivel $18 \mathrm{~b}$, que como los niveles analizados en el anterior estudio, se encuendra en la escala temporal coincidente con la transición del Paleolítico Medio al Superior, en concreto con el Auriñacience de Transición del yacimiento arqueológico Cueva de El Castillo.

A los fines operativos de este estudio, se consideran como microdesechos líticos a todos los fragmentos menores de $1 \mathrm{~cm}^{2}$ dentro de una amplia categoría propuesta por Fish (1981) que incluye a todos los subproductos de preparación del núcleo, etapas de elaboración de útiles y los resultantes de la modificación y mantenimiento durante la vida útil de los artefactos (Fish 1981:374).

\subsection{Objetivos}

El objetivo general de este trabajo es la identificación de actividades relacionadas con el subsistema de producción lítico (o cadena operativa) llevadas a cabo en El Castillo durante la manufactura, reciclado, mantenimiento y el uso de instrumentos (Peretti y Mingo 2000). Igualmente, nos hemos planteado otros objetivos específicos:

1. Analizar y discutir el aprovechamiento de las diferentes materias primas líticas.

2. Analizar la variabilidad en la distribución espacial (horizontal y vertical) de los microdesechos líticos a nivel intrasitio.

3. Contribuir a la mejor comprensión de la dinámica de formación de este yacimiento.

4. Aportar un nuevo caso de estudio que sirva de modelo en el análisis de los microdesechos líticos.

\section{ANTECEDENTES}

\subsection{Cueva de El Castillo}

Esta cueva se localiza en Puente Viesgo (Cantabria, España), 30 kilómetros al sur de Santander, concretamente se encuentra en un monte calcáreo de origen Namuriense (Carbonífero Superior) llamado del Castillo (Figura 1).

El yacimiento se localiza en el área externa de una amplia red de complejos cársticos, correspondiendo con una amplia sala cortada en su extremo

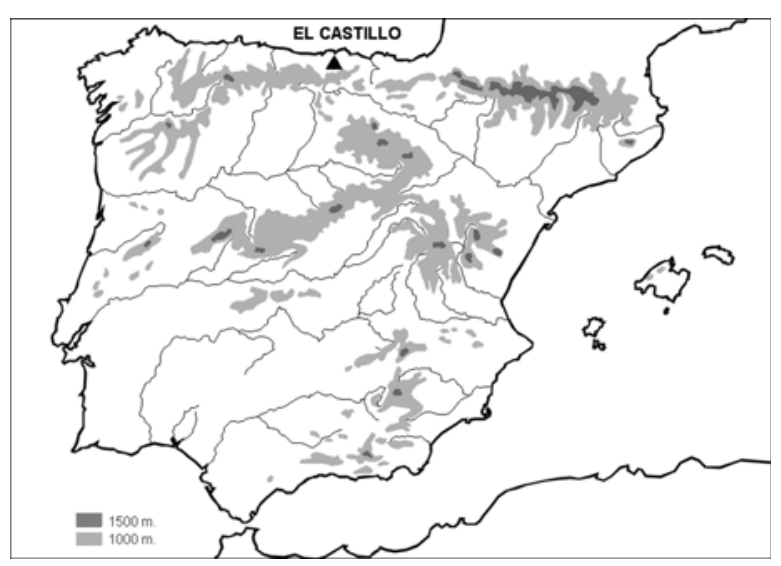

Fig. 1. Localización del yacimiento Cueva de El Castillo (Puente Viesgo, Cantabria).

por la evolución de la vertiente dando lugar a una gran cavidad con un vestíbulo de sección pseudo rómbica de 18 a $20 \mathrm{~m}$ de ancho y una altura visible actual alrededor de los $20 \mathrm{~m}$; se debe destacar que en el pasado estuvo prácticamente colmatada por sedimentos (Cabrera Valdés et al. 1993). La secuencia estratigráfica de la cueva de El Castillo representa una de las más amplias conocidas en la Península Ibérica, albergando una sucesión completa de ocupaciones paleolíticas a lo largo de 26 capas, en donde se alternan las estériles con las de ocupación humana. Se determinaron los siguientes niveles culturales: 2 niveles con Paleolítico Medio Antiguo, un nivel Achelense Superior, dos niveles Musterienses, dos niveles Auriñacienses (entre ellos el nivel 18b, denominado Auriñaciense de Transición), dos del Perigordiense Superior, uno del Solutrense Medio, tres niveles Magdalenienses $\mathrm{y}$, por último, un Aziliense.

En cuanto a las características del nivel $18 \mathrm{~b}$ hay que decir que en la intersección con el nivel 18c se encuentran arcillas pardas ricas en materia orgánica englobando cantos y bloques de caliza dispersos o en concentraciones irregulares; ambos niveles se caracterizan por presentar abundante materia orgánica en detrimento de elementos detríticos calcáreos. La estratigrafía es masiva e irregular, tendiendo a paralela, con un ligero buzamiento hacia el interior. El análisis de la sedimentación del nivel $18 \mathrm{~b}$ es complejo debido a las transformaciones antrópicas. El nivel 18b se sitúa en la zona exterior y presenta arcillas y algunos bloques que se apoyan sobre el contrafuerte externo del nivel 19. Al final de la sedimentación de este nivel, se produce un derrumbamiento de bloques de la bóveda, con un con- 
siderable retroceso del techo de la entrada y una importante modificación de la morfología del yacimiento. Todo esto origina un nuevo contrafuerte externo que condicionará la zona de ocupación desplazándola y retrotrayéndola hacia el interior. (Cabrera Valdés et al. 1993; Cabrera Valdés et al. 2001). La interpretación climática del nivel 18 ha sido considerada como el inicio de una pulsación fría no demasiado rigurosa.

La industria lítica hallada en el nivel 18 (tanto $18 \mathrm{~b}$ como $18 \mathrm{c}$ ) es bastante homogénea y se caracteriza principalmente por poseer una porcentaje importante de raspadores. Estos datos concuerdan con los obtenidos para estos niveles en las excavaciones que Obermaier realizó en este yacimiento a principios de siglo. La industria ósea esta representada por numerosos elementos descubiertos en la base del nivel 18c: extremo distal de un cincel que contiene una serie rítmica de trazos horizontales (marcas de caza), un extremo distal de una azagaya en asta y fragmentos de asta que se podrían relacionar con la fabricación de azagayas. Este tipo de materiales están ya presentes en la colección antigua (Cabrera Valdés et al. 1996). Es reseñable también el hallazgo reciente en el nivel $18 \mathrm{~b}$ de un fragmento de hueso hioides que presenta un grabado naturalista, quizá una cierva, y de un fragmento de plaqueta de arenisca grabada con unas líneas profundas en U (Cabrera Valdés et al. 2001).

Se dispone de cinco dataciones por C14 AMS para el nivel 18b, obtenidas en dos laboratorios diferentes, Tucson (Cabrera y Bischoff 1989) y Oxford (Hedges et al. 1994). Todas se sitúan en torno al 38.500 BP. Estas fechas, junto con las obtenidas para el nivel 18c (en torno al $40.000 \mathrm{BP}$ ) podrían confirmar la existencia de un Auriñaciense anterior al Chatelperroniense.

En términos culturales, este yacimiento, durante el período de ocupación coincidente con el nivel 18, ha sido considerado como un sitio de agregación siendo su tendencia funcional como campamento base (Bernaldo de Quirós y Cabrera Valdés 1996).

\subsection{Breves apuntes sobre las investigaciones de microdesechos líticos}

Los estudios referidos al registro arqueológico de características microscópicas se vienen desarrollando desde la década de los setenta. Durante todo este periodo hasta la actualidad estos trabajos han profundizado en distintas vías de análisis entre las que se pueden destacar: identificación de patrones de organización espacial de comportamiento dentro de las estructuras de un sitio, reconstrucción de áreas de actividad e interpretación funcional de sitios, inferencias acerca del carácter y la magnitud de las alteraciones post-ocupacionales, determinación de técnicas de talla lítica, diferenciación de desechos primarios y secundarios, descubrimiento y detección de sitios en condiciones de baja visibilidad y reconstrucción de los ambientes deposicionales (Clark 1986; Dunnell y Stein 1989; Fladmark 1982; Hassan 1978; Hull 1987; Patterson y Sollberger 1978; Peretti y Mingo 2000; Sullivan y Rozen 1985; Schiffer 1972).

Una gran cantidad de investigadores reconocen la importancia del análisis de los restos de talla (Ericson 1984; Shott 1994; Sullivan y Rozen 1985). En concreto, los microdesechos se distinguen de otros artefactos ya que no son objeto frecuente de transporte sino que una vez depositados, a priori, permanecen in situ. Estos restos originados a lo largo de las diferentes fases de la cadena operativa son retenidos en las matrices de los sitios arqueológicos y pueden ser analizados desde aspectos funcionales al tiempo que se pueden detectar posibles efectos generados por agentes naturales (Clark 1986; Cowan 1999; Dunnell y Stein 1989; Fladmark 1982; Hull 1987; Patterson y Sollberger 1978; Peretti 1997; Peretti y Mingo 2000).

En este sentido, Morrow (1996) sugiere que los artefactos manufacturados en un yacimiento y posteriormente transportados quedarán separados de los microdesechos derivados de su manufactura. En esta situación, los microdesechos que quedan en el yacimiento inicial forman un "Ghost" con respecto a los artefactos transportados. Del mismo modo, los útiles que han entrado en un yacimiento y que no están acompañados de los microdesechos derivados de su elaboración formarán "Orphans".

Las distintas etapas de la secuencia de reducción lítica (i.e. manufactura, uso, modificación y mantenimiento opcional) pueden ser identificadas mediante una alta densidad de microdesechos líticos, comparados con las densidades de macrodesechos y útiles (Hull 1987:773; Morrow 1996:358). Por lo tanto, la comparación de objetos pequeños y grandes mediante diagramas de distribución, teniendo en cuenta el tipo de materias primas y las categorías de manufactura de utensilios, permite la identificación de los procesos de formación de sitios específicamente culturales (Morrow 1996; Schiffer 1983). 


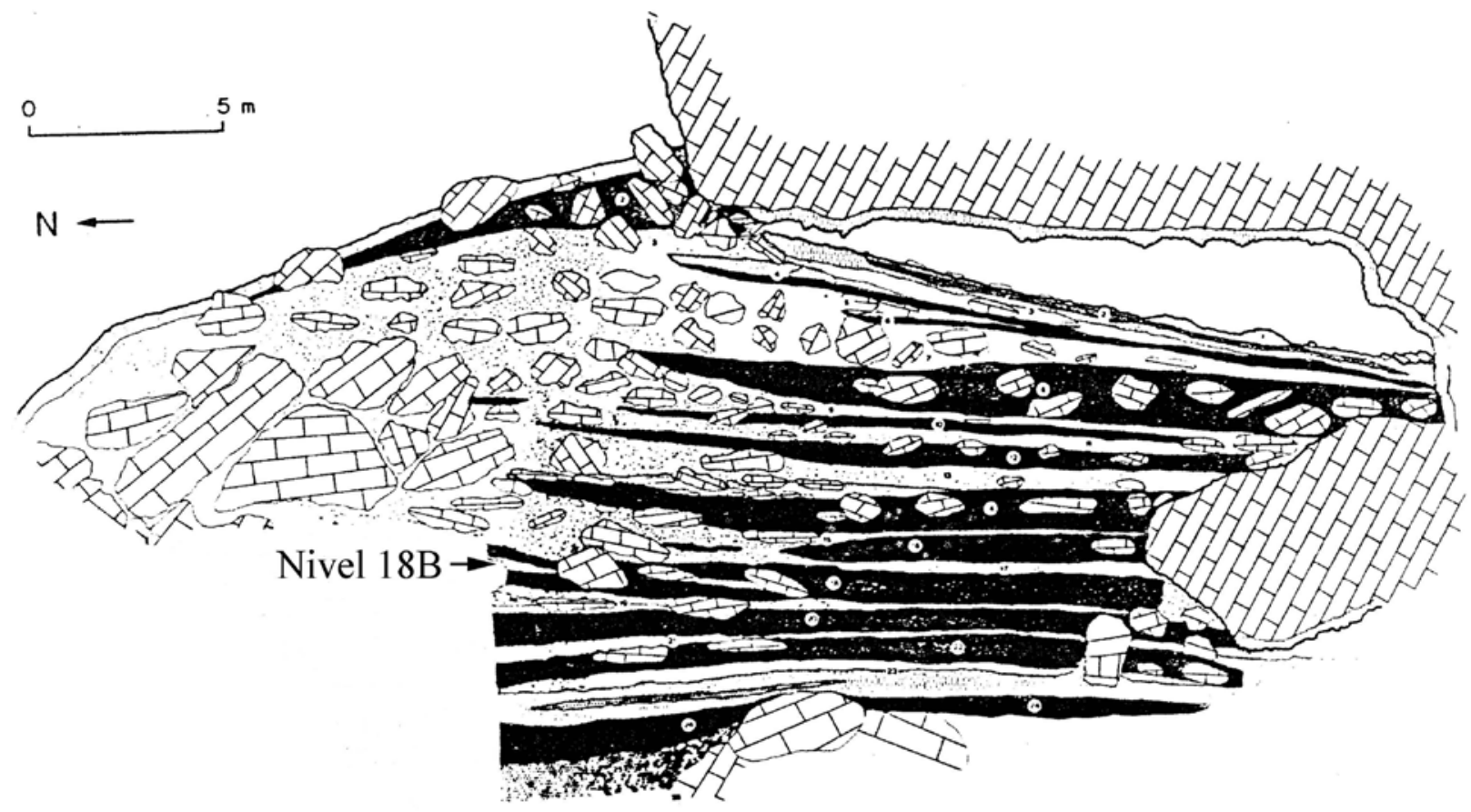

Fig. 2. Estratigrafía general del yacimiento.

En cuanto a las distribuciones espaciales, la mayoría de los investigadores insisten en la realización de este tipo de análisis a nivel intrasitio para esclarecer disposiciones no aleatorias de elementos. Desde el trabajo pionero de Ascher (1961) los investigadores vienen tomando conciencia de la importancia que tienen estos estudios y los procesos de formación de los depósitos arqueológicos como un medio para comprender mejor la dinámica del comportamiento humano en el pasado.

\section{CONSIDERACIONES METODOLÓGICAS Y TÉCNICAS}

En este trabajo hemos continuado con la metodología empleada en el anterior estudio (Peretti y Mingo 2000) siguiendo las líneas analíticos y códigos propuestos por Aschero (1975, 1983), Bellelli et al., (1985-1987) y Nami (1991a y b). La identificación de los productos de talla se realizó según la presencia de ciertos atributos. Así, se consideraron como lascas a aquellos artefactos producidos por la fractura intencional de formas base donde se diferencian atributos tecnológicos tales como talón, bulbo, estrías, etc.

Los microdesechos líticos analizados en este estudio fueron obtenidos durante las excavaciones de las cuadrículas G 15, H 15, I 13, I 14, I 15, J 13, J 14, J 15, K 12, K 13, K 14, K 15, K 16, L 11, L 12, L 13, L 14, L 15 y M12 y constituyen un total de 11.984 piezas.

Los materiales de planta, por una parte, fueron registrados tridimensionalmente mientras que el material de dimensiones muy pequeñas, por otra, fue recogido mediante el uso de cribas de malla fina de $0,04 \mathrm{~cm}$. Una vez en el laboratorio se llevo a cabo la separación de los diferentes materiales contenidos en las bolsas (i.e. óseos, coprolitos, carbones, líticos). Posteriormente, se realizaron los siguientes pasos:

- Análisis macroscópico y microscópico de los microdesechos con objeto de registrar los atributos tecnológicos. Para la observación microscópica de los elementos se usó un microscopio estereoscopico Kyowa SDZ. Con un calibre manual se tomaron medidas de largo, ancho y espesor a fin de determinar un máximo y un mínimo de tamaño. La información recogida fue almacenada en la ficha de análisis propuesta por Bellelli et al (1985-1987).

- Utilización de las variables propuestas en el programa DELCO (Desechos Líticos Computarizados), metodología que hace posible el análisis del conjunto de atributos controlados (Bellelli et al.. 1985-1987).

- Utilización del programa EXCEL 2000 (Mi- 
crosoft Corporation) en la elaboración de tablas y figuras.

Las variables y atributos analizados en los microdesechos líticos son los siguientes:

- Materia prima: identificación de los diferentes tipos de materia prima existentes en el yacimiento.

- Estado: mediante esta categoría se realiza una primera clasificación de los desechos de talla a partir de la presencia o ausencia de fragmentación (Bellelli et al., 1985-1987). En el mismo pueden intervenir diversos factores como, por ejemplo, la calidad de la materia prima, los accidentes de talla, el pisoteo, el transporte, el uso, etc. Dentro de esta categoría hemos definido cuatro tipos de microdesechos: lasca entera, lasca fracturada con talón, lasca fracturada sin talón y lasca indiferenciada. A éste último tipo corresponden aquellos microdesechos que a pesar de proceder de la talla lítica presentan un grado de fracturación tan elevado o una morfología que no permite diferenciar su cara dorsal de la bulbar.

- Tipo: es un indicador tecno-morfológico que permite inferir en que etapa de la secuencia de producción lítica se encuentran los microdesechos. En esta categoría hemos identificado lascas primarias, secundarias, de arista, angulares, planas, de reactivación directa (en este grupo se incluyen las lascas procedentes del reavivado de los filos de los útiles) e indiferenciadas (a este segmento corresponden las lascas que aún presentando atributos claros no se las puede englobar en otras categorías porque presentan fracturas, de modo que no se puede reconocer de una forma segura el tipo de lasca; así, todas aquellas lascas fracturadas cuya cara dorsal fuera plana, de arista o con presencia de corteza han integrado las indiferenciadas) (Aschero 1975, 1983).

- Módulo de Longitud-Anchura y Espesor: configuran dimensiones relativas de largo y ancho utilizando el gráfico de Bagolini (1968), modificado por Aschero $(1975,1983)$, y una medida absoluta como el espesor. Estos atributos se utilizan específicamente en la evaluación de las distintas etapas de manufactura presentes.

- Las características de talón y bulbo son indicadoras de las técnicas de percusión y presión aplicadas con percutores blandos y duros (Baumler 1985; Nami 1991a ; Patterson y Sollberger 1978).

- Presencia de corteza: esta variable proporciona información sobre el grado de reducción alcanzada en cada etapa de la manufactura (Aschero 1975, 1983; Cowan 1999; Nash 1996).
Es necesario añadir que, en relación a los atributos, la no existencia de un grado de certeza absoluta acerca de los mismos nos hace considerarlos ausentes.

Para identificar los procesos de formación cultural de El Castillo, se implementaron diagramas de distribución sobre la base de los análisis realizados en los microdesechos por cuadrícula y sector en las áreas excavadas. En cada cuadrícula se analizaron la frecuencia de las distintas materias primas, con el fin de identificar patrones que pudieron alterar la distribución y el estado de los microdesechos líticos, y así poder evaluar el comportamiento de diferentes matrices con respecto a: migración, dispersión y alteración morfológica de elementos líticos contenidas en ellas.

Para el estudio de los procesos de formación y con el fin de poder determinar los factores culturales y/o naturales que hayan podido modificar el depósito arqueológico se consideraron en este trabajo los aportes efectuados por los:

A. Estudios de arqueología experimental (i.e. Nami 1991 a y b, 1992 a y b; Maíllo Fernández 1998; Patterson y Sollberger 1978; Peretti y Curtoni 1999; entre otros).

B. Estudios de etno-arqueología (i.e. Binford 1978; Clark 1986; Gould 1979; entre otros)

C. Estudios específicos de micromamíferos (Bocek 1986; Gómez 1996; entre otros)

D. Acción del pisoteo (Clark 1986; McBrearty et al., 1998; Villa 1982; entre otros)

E. Acción de las pendientes y del agua (Cahen y Moeyersons 1977; Peretti y Curtoni 1999; Schiffer 1987; entre otros).

\section{RESULTADOS}

\section{Materias primas líticas}

Hemos podido identificar en el registro de microdesechos líticos los siguientes tipos de materia prima: arenisca, cuarcita, calcita, caliza, cuarzo, limonita, sílex y ofita, y un número de rocas que no han podido ser determinadas y que han sido calificadas como indiferenciadas. La calcita no se ha contabilizado en el cómputo general ya que el estado descompuesto en que aparece hace prácticamente imposible discernir si su origen es natural o antrópico. La caliza, por el contrario, si ha sido tenida en cuenta a efectos de cómputo puesto que esta materia prima fue aportada al yacimiento, si bien, como en la cal- 
cita, su estado alterado y en descomposición tampoco nos ha permitido diferenciar con claridad sus atributos, de esta forma en el resto de tablas no ha sido tenida en cuenta. Las materias primas identificadas en el nivel 18b fueron definidas por Cabrera Valdés et al., (1996). El sílex aparece muy alterado (desilificado). Con respecto a la cuarcita se presenta de la siguiente manera:

Cuarcita 1: en cantos pequeños, de colores variados y de grano muy fino.

Cuarcita 2: de grano fino, de cantos mayores y de color verde.

Cuarcita 3: de grano fino muy compacto con inclusiones de minúsculas puntuaciones de manganeso de color gris opaco.

Respecto del total de microdesechos recuperados en el sitio $(\mathrm{N}=15385)$ se observa que la cuarcita 1 (C1), con un porcentaje del $55,87 \%$, es la más representada, la caliza alcanza el $22,11 \%$, y a ésta le siguen el sílex con $11,36 \%$, y el cuarzo con 5,90 $\%$. El resto de materias primas no superaría el $2 \%$ (tabla 1).

\begin{tabular}{|l|r|r|}
\hline \multicolumn{1}{|c|}{ Materia prima } & \multicolumn{1}{c|}{ N } & \multicolumn{1}{c|}{ \% } \\
\hline Arenisca & 70 & 0,45 \\
\hline Cuarcita 1 & 8.595 & 55,87 \\
\hline Cuarcita 2 & 104 & 0,68 \\
\hline Cuarcita 3 & 261 & 1,70 \\
\hline Caliza & 3.401 & 22,11 \\
\hline Cuarzo & 908 & 5,90 \\
\hline Indiferenciada & 89 & 0,58 \\
\hline Limonita & 26 & 0,17 \\
\hline Ofita & 183 & 1,19 \\
\hline Sílex & 1.748 & 11,36 \\
\hline Total general & $\mathbf{1 5 . 3 8 5}$ & $\mathbf{1 0 0 , 0 0}$ \\
\hline
\end{tabular}

Tab. 1. Materias primas líticas de la muestra general.

\section{Estado de Fragmentación}

Se han considerado un total de 11.984 microdesechos, al no poderse tener en cuenta a las calizas (Tab. 2). Se observa a las lascas enteras como las más numerosas $(37,38 \%)$, inmediatamente después y con un porcentaje muy similar están las indiferenciadas $(36,27 \%)$. Las lascas fracturadas sin talón

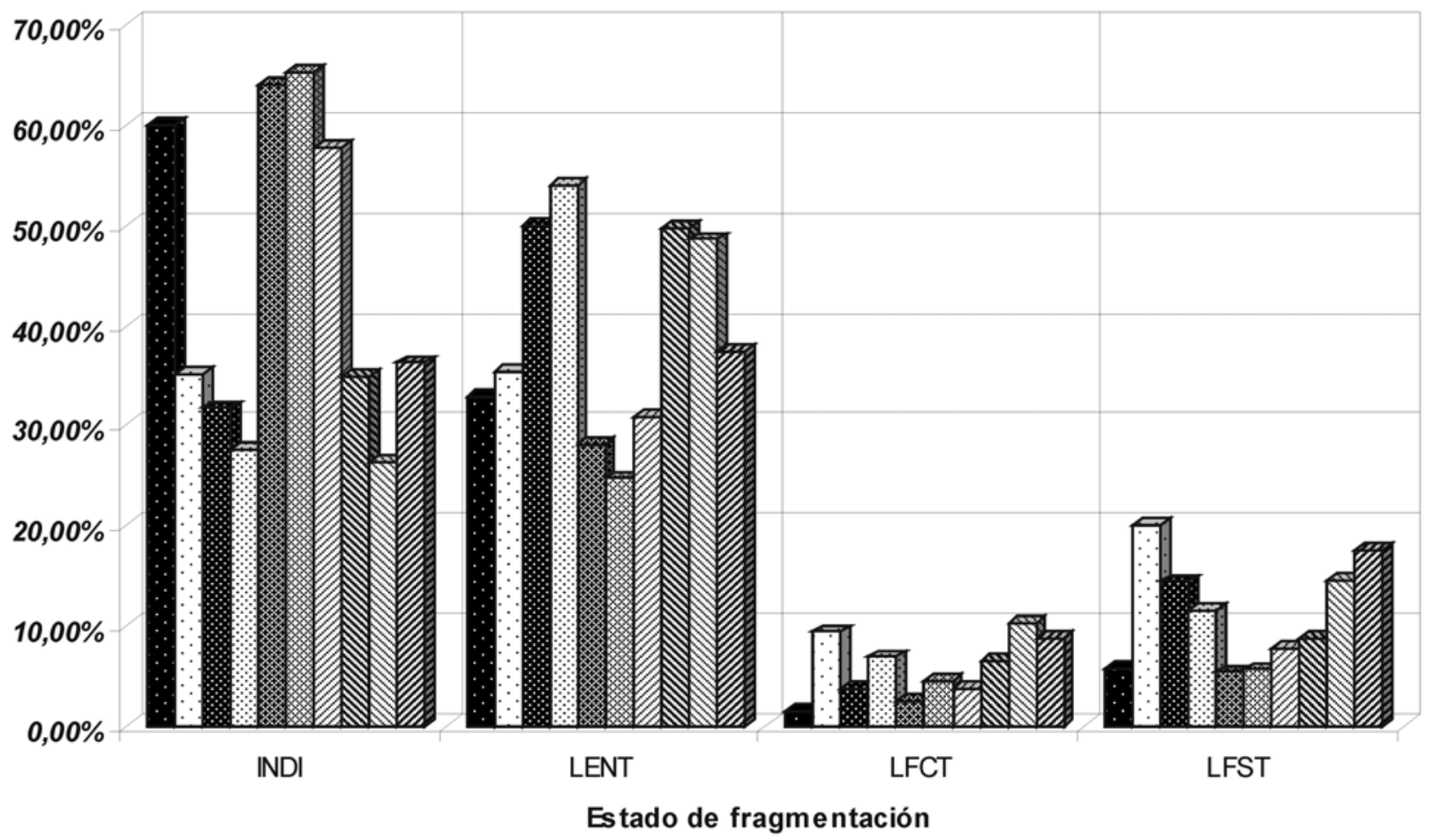

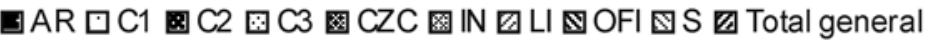

Fig. 3. Estado por materia prima. Referencias: INDI: indiferenciada, LENT: lasca entera, LFCT: lasca fracturada con talón, LFST: lasca fracturada sin talón. 
$(17,58 \%)$ y las fracturadas con talón $(8,77 \%)$ no llegan entre ambas al $30 \%$.

\begin{tabular}{|l|c|r|}
\hline \multicolumn{1}{|c|}{ Estado } & N & \% \\
\hline Indiferenciada & 4.346 & 36,27 \\
\hline Entera & 4.480 & 37,38 \\
\hline Fracturada con talón & 1.051 & 8,77 \\
\hline Fracturada sin talón & 2.107 & 17,58 \\
\hline Total general & $\mathbf{1 1 . 9 8 4}$ & $\mathbf{1 0 0 , 0 0}$ \\
\hline
\end{tabular}

Tab. 2. Estado de los microdesechos.

El análisis del estado de fragmentación por materia prima (Fig. 3) nos hace constatar que la frecuencia de lascas enteras es muy alta en la cuarcita $3(54,02 \%)$, cuarcita $2(50 \%)$, ofita $(49,73 \%)$ y sílex $(48,57 \%)$ por este orden; todas ellas en torno al $50 \%$ del total de las mismas. En cambio, las indiferenciadas predominan en arenisca $(60 \%)$, cuarzo $(64,10 \%)$ y limonita $(57,69 \%)$.

\section{Tipo de lasca}

Se han analizado 7638 elementos del total no habiéndose considerado las indiferenciadas (tabla 3). De estos resultados se puede observar que las lascas angulares dominan ampliamente el conjunto con un $57,34 \%$, seguidas por las planas con un $15,11 \%$ y las indiferenciadas con un $14,57 \%$. El resto de las categorías, no supera el $5 \%$.

La presencia de corteza (que engloba tanto a lascas primarias y secundarias como a las que tienen talón cortical o algo de corteza en su cara dorsal) representa con respecto al resto del conjunto el $13,31 \%$ (tabla 4). En este sentido, la materia prima que presenta mayor frecuencia de corteza (tabla 5) es la cuarcita 1 con un $83,82 \%$, el cuarzo tiene un $10,28 \%$ y el resto no supera el $3 \%$.

\begin{tabular}{|l|r|r|}
\hline \multicolumn{1}{|c|}{ Tipo lasca } & N & \% \\
\hline Angular & 4.379 & 57.34 \\
\hline Arista & 250 & 3,27 \\
\hline Indiferenciada & 1.113 & 14,57 \\
\hline Plana & 1.154 & 15,11 \\
\hline Primaria & 179 & 2,34 \\
\hline Reactivación directa & 347 & 4,54 \\
\hline Secundaria & 216 & 2,83 \\
\hline Total general & $\mathbf{7 . 6 3 8}$ & $\mathbf{1 0 0 , 0 0}$ \\
\hline
\end{tabular}

Tab. 3. Tipo de lasca.

\begin{tabular}{|l|r|r|}
\hline \multicolumn{1}{|c|}{ Corteza } & N & \multicolumn{1}{c|}{ \% } \\
\hline Presencia & 1.595 & 13,31 \\
\hline Ausencia & 10.389 & 86,69 \\
\hline Total & $\mathbf{1 1 . 9 8 4}$ & $\mathbf{1 0 0 , 0 0}$ \\
\hline
\end{tabular}

Tab. 4. Representación de corteza.

\begin{tabular}{|l|r|r|}
\hline \multicolumn{1}{|c|}{ Materia prima } & N & \% \\
\hline Arenisca & 4 & 0,25 \\
\hline Cuarcita 1 & 1.337 & 83,82 \\
\hline Cuarcita 2 & 12 & 0,75 \\
\hline Cuarcita 3 & 27 & 1,69 \\
\hline Cuarzo & 164 & 10,28 \\
\hline Indiferenciada & 7 & 0,44 \\
\hline Limonita & 2 & 0,13 \\
\hline Ofita & 9 & 0,56 \\
\hline Sílex & 33 & 2,07 \\
\hline Total general & $\mathbf{1 . 5 9 5}$ & $\mathbf{1 0 0 , 0 0}$ \\
\hline
\end{tabular}

Tab. 5. Materia prima que presenta reserva de corteza.

\section{Módulo longitud anchura}

Según lo expuesto por Bellelli et al. (1985-1987) el análisis de esta variable tan solo se puede llevar a cabo sobre los microdesechos enteros (Tabla 6). De un total de 4480 elementos se observa un predominio del rango mediano normal $(30,65 \%)$ y corto $(24,29 \%)$, superando entre ambos el $55 \%$ del conjunto total de elementos analizados. A éstos dos le siguen el corto muy ancho $(17,30 \%)$, el mediano alargado $(13,24 \%)$ y corto anchísimo $(7,34 \%)$. Por último, estarían representados con una frecuencia baja con respecto al resto los módulos laminares: laminar normal $(5,85 \%)$, laminar angosto $(1,29 \%)$ y laminar muy angosto $(0,04 \%)$.

\begin{tabular}{|l|r|r|}
\hline \multicolumn{1}{|c|}{ Módulo L x A } & N & \% \\
\hline Laminar muy angosto & 2 & 0,04 \\
\hline Laminar angosto & 58 & 1,29 \\
\hline Laminar normal & 262 & 5,85 \\
\hline Mediano alargado & 593 & 13,24 \\
\hline Mediano normal & 1.373 & 30,65 \\
\hline Corto & 1.088 & 24,29 \\
\hline Corto muy ancho & 775 & 17,30 \\
\hline Corto anchísimo & 329 & 7,34 \\
\hline Total general & $\mathbf{4 . 4 8 0}$ & $\mathbf{1 0 0 , 0 0}$ \\
\hline
\end{tabular}

Tab. 6. Módulo de longitud-anchura.

Completando esta información, presentamos una escala para tres rangos de espesores (Figura 4), donde se puede comprobar el dominio del rango 0,005-0,3 (el más delgado) sobre los otros dos. 


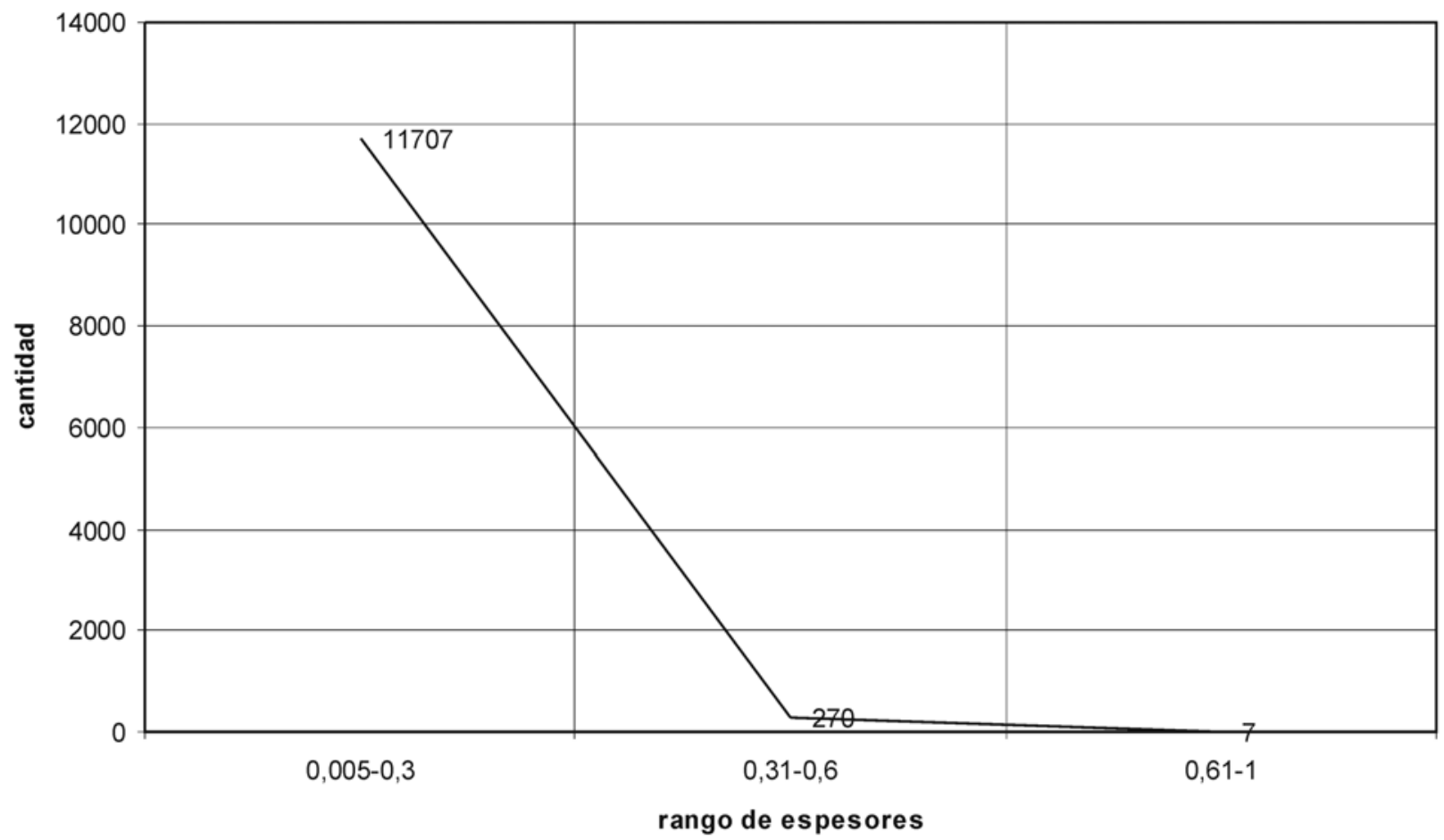

Fig. 4. Intervalos de espesores.

\section{Talones y bulbos}

En nuestro estudio, a diferencia del trabajo anterior de Peretti y Mingo (2000), tan solo hemos analizado los bulbos de las lascas enteras o fracturadas que también conservan su talón. De este modo, de un total de 11.984 microdesechos se han podido identificar 5.531 con talón (tabla 7). Los talones preparados son los más frecuentes y el más representado es el liso $(62,76 \%)$, seguido por el filiforme $(19,26 \%)$, el puntiforme $(5,73 \%)$, diedro $(2,57 \%) y$, finalmente, el facetado $(1,12 \%)$. El talón indiferenciado $(1,72 \%)$ es aquel que presenta una fractura, de tal forma que es imposible aseverar totalmente su tipología. Para los talones no preparados (corticales) se aprecia una baja presencia $(6,85 \%)$ con respecto al total.

\begin{tabular}{|l|r|r|}
\hline \multicolumn{1}{|c|}{ Tipo de talón } & N & \% \\
\hline Cortical & 379 & 6,85 \\
\hline Diedro & 142 & 2,57 \\
\hline Facetado & 62 & 1,12 \\
\hline Filiforme & 1.065 & 19,26 \\
\hline Indiferenciado & 95 & 1,72 \\
\hline Liso & 3.471 & 62,76 \\
\hline Puntiforme & 317 & 5,73 \\
\hline Total general & $\mathbf{5 . 5 3 1}$ & $\mathbf{1 0 0 , 0 0}$ \\
\hline
\end{tabular}

Tab. 7. Tipo de talones.
En relación a los bulbos (tabla 8), el más frecuente es el difuso $(55,04 \%)$ seguido del indiferenciado $(34,03 \%)$ y el pronunciado $(10,94 \%)$.

\begin{tabular}{|l|r|r|}
\hline \multicolumn{1}{|c|}{ Tipo de bulbo } & N & \% \\
\hline Difuso & 3.044 & 55,04 \\
\hline Indiferenciado & 1.882 & 34,03 \\
\hline Pronunciado & 605 & 10,94 \\
\hline Total general & $\mathbf{5 . 5 3 1}$ & $\mathbf{1 0 0 , 0 0}$ \\
\hline
\end{tabular}

Tab. 8. Tipo de bulbos.

Para profundizar en el análisis se han combinado estos atributos, estudiando cada materia prima de forma individualizada. No fueron consideradas en este análisis por su bajo número (ya que sobredimensionarían los resultados) la arenisca, el cuarzo, las indeferenciadas, la limonita y la ofita. La cuarcita 2 y la cuarcita 3 se decidió analizarlas para comparar sus resultados con la cuarcita 1 ya que, presumiblemente, podrían tener propiedades de talla parecidas.

\section{Lascas de reactivación}

Del total de microdesechos ( $\mathrm{N}=11984)$ se pudieron reconocer 341 lascas de reactivación directas. 


\begin{tabular}{|c|c|c|c|c|c|c|c|c|}
\hline \multirow{2}{*}{$\begin{array}{l}\text { Tipo de bulbo } \\
\text { Tipo de talón }\end{array}$} & \multicolumn{2}{|c|}{ Difuso } & \multicolumn{2}{|c|}{ Indiferenciado } & \multicolumn{2}{|c|}{ Pronunciado } & \multicolumn{2}{|c|}{ Total general } \\
\hline & $\mathbf{N}$ & $\%$ & $\mathbf{N}$ & $\%$ & $\mathbf{N}$ & $\%$ & $\mathbf{N}$ & $\%$ \\
\hline Cortical & 157 & 4,08 & 130 & 3,38 & 25 & 0,65 & 312 & 8,11 \\
\hline Diedro & 48 & 1,25 & 25 & 0,65 & 21 & 0,55 & 94 & 2,44 \\
\hline Facetado & 26 & 0,68 & 8 & 0,21 & 6 & 0,16 & 40 & 1,04 \\
\hline Filiforme & 478 & 12,43 & 235 & 6,11 & 57 & 1,48 & 770 & 20,02 \\
\hline Indiferenciado & 54 & 1,40 & 25 & 0,65 & 3 & 0,08 & 82 & 2,13 \\
\hline Liso & 1.218 & 31,66 & 882 & 22,93 & 273 & 7,10 & 2.373 & 61,68 \\
\hline Puntiforme & 106 & 2,76 & 57 & 1,48 & 13 & 0,34 & 176 & 4,57 \\
\hline Total & 2.087 & 54,25 & 1.362 & 35,40 & 398 & 10,35 & 3.847 & 100,00 \\
\hline
\end{tabular}

Tab. 9. Cantidad y porcentaje de talones y bulbos en cuarcita 1 .

\begin{tabular}{|l|c|c|c|c|c|r|r|r|}
\hline \multicolumn{1}{|c|}{ Tipo de bulbo } & \multicolumn{2}{c|}{ Difuso } & \multicolumn{2}{c|}{ Indiferenciado } & \multicolumn{2}{c|}{ Pronunciado } & \multicolumn{2}{c|}{ Total general } \\
\hline \multicolumn{1}{|c|}{ Tipo de talón } & $\mathbf{N}$ & $\mathbf{\%}$ & $\mathbf{N}$ & $\mathbf{\%}$ & $\mathbf{N}$ & $\mathbf{\%}$ & \multicolumn{1}{c|}{$\mathbf{N}$} & $\mathbf{\%}$ \\
\hline Cortical & 3 & 5,36 & 1 & 1,79 & 1 & 1,79 & 5 & 8,93 \\
\hline Facetado & & & & & 1 & 1,79 & 1 & 1,79 \\
\hline Filiforme & 2 & 3,57 & & & & & 2 & 3,57 \\
\hline Liso & 31 & 55,36 & 10 & 17,86 & 6 & 10,71 & 47 & 83,93 \\
\hline Puntiforme & & & 1 & 1,79 & & & 1 & 1,79 \\
\hline Total & $\mathbf{3 6}$ & $\mathbf{6 4 , 2 9}$ & $\mathbf{1 2}$ & $\mathbf{2 1 , 4 3}$ & $\mathbf{8}$ & $\mathbf{1 4 , 2 9}$ & $\mathbf{5 6}$ & $\mathbf{1 0 0 , 0 0}$ \\
\hline
\end{tabular}

Tab. 10. Cantidad y porcentaje de talones y bulbos en cuarcita 2 .

\begin{tabular}{|l|c|c|c|c|c|r|r|r|}
\hline \multicolumn{1}{|c|}{ Tipo de bulbo } & \multicolumn{2}{c|}{ Difuso } & \multicolumn{2}{c|}{ Indiferenciado } & \multicolumn{2}{c|}{ Pronunciado } & \multicolumn{2}{c|}{ Total general } \\
\hline \multicolumn{1}{|c|}{ Tipo de talón } & $\mathbf{N}$ & $\mathbf{\%}$ & $\mathbf{N}$ & $\mathbf{\%}$ & $\mathbf{N}$ & $\mathbf{\%}$ & $\mathbf{N}$ & $\boldsymbol{\%}$ \\
\hline Cortical & 6 & 3,77 & 3 & 1,89 & 1 & 0,63 & 10 & 6,29 \\
\hline Diedro & 3 & 1,89 & & & 2 & 1,26 & 5 & 3,14 \\
\hline Facetado & & & 1 & 0,63 & & & 1 & 0,63 \\
\hline Filiforme & 7 & 4,40 & 7 & 4,40 & & & 14 & 8,81 \\
\hline Liso & 66 & 41,51 & 52 & 32,70 & 10 & 6,29 & 128 & 80,50 \\
\hline Puntiforme & & & 1 & 0,63 & & & 1 & 0,63 \\
\hline Total & $\mathbf{8 2}$ & $\mathbf{5 1 , 5 7}$ & $\mathbf{6 4}$ & $\mathbf{4 0 , 2 5}$ & $\mathbf{1 3}$ & $\mathbf{8 , 1 8}$ & $\mathbf{1 5 9}$ & $\mathbf{1 0 0 , 0 0}$ \\
\hline
\end{tabular}

Tab. 11. Cantidad y porcentaje de talones y bulbos en cuarcita 3 .

\begin{tabular}{|c|c|c|c|c|c|c|c|c|}
\hline \multirow{2}{*}{$\begin{array}{c}\text { Tipo de bulbo } \\
\text { Tipo de talón }\end{array}$} & \multicolumn{2}{|c|}{ Difuso } & \multicolumn{2}{|c|}{ Indiferenciado } & \multicolumn{2}{|c|}{ Pronunciado } & \multicolumn{2}{|c|}{ Total general } \\
\hline & $\mathbf{N}$ & $\%$ & $\mathbf{N}$ & $\%$ & $\mathbf{N}$ & $\%$ & $\mathbf{N}$ & $\%$ \\
\hline Cortical & 4 & 0,39 & 1 & 0,10 & & 0,00 & 5 & 0,49 \\
\hline Diedro & 23 & 2,23 & 12 & 1,17 & 3 & 0,29 & 38 & 3,69 \\
\hline Facetado & 14 & 1,36 & & 0,00 & 2 & 0,19 & 16 & 1,55 \\
\hline Filiforme & 140 & 13,60 & 51 & 4,95 & 42 & 4,08 & 233 & 22,62 \\
\hline Indiferenciado & 7 & 0,68 & 4 & 0,39 & & 0,00 & 11 & 1,07 \\
\hline Liso & 327 & 31,75 & 177 & 17,18 & 95 & 9,22 & 599 & 58,16 \\
\hline Puntiforme & 69 & 6,70 & 47 & 4,56 & 12 & 1,17 & 128 & 12,43 \\
\hline Total & 584 & 56,70 & 292 & 28,35 & 154 & 14,95 & 1.030 & 100,00 \\
\hline
\end{tabular}

Tab. 12. Cantidad y porcentaje de talones y bulbos en sílex.

\begin{tabular}{|l|r|r|}
\hline \multicolumn{1}{|c|}{ Materia prima } & N & \multicolumn{1}{c|}{ \% } \\
\hline Cuarcita 1 & 241 & 69,46 \\
\hline Cuarcita 2 & 3 & 0,86 \\
\hline Cuarcita 3 & 10 & 2,88 \\
\hline Cuarzo & 18 & 5,19 \\
\hline Ofita & 4 & 1,15 \\
\hline Sílex & 71 & 20,46 \\
\hline Total general & $\mathbf{3 4 7}$ & $\mathbf{1 0 0 , 0 0}$ \\
\hline
\end{tabular}

Tab. 13. Lasca de reactivación directa (RD) por materia prima. 
En lo relativo a la materia prima, es en la cuarcita 1 donde más lascas de este tipo se han registrado $(69,46 \%)$, le siguen el sílex $(20,46 \%)$, el cuarzo $(5,19 \%)$, la cuarcita $3(2,88 \%)$, la ofita $(1,15 \%)$, y por último la cuarcita $2(0,86 \%)$.

\section{Microdesechos quemados}

Hemos registrado 5 microdesechos de sílex quemados y 16 microdesechos rubefactados cuya materia prima nos ha sido imposible identificar con total certitud por lo que se han considerado como materia prima indiferenciada. Su distribución espacial no refleja la existencia de concentraciones de los mismos, por el contrario, se localizan bastante dispersos.

\section{Distribuciones espaciales}

El diagrama de distribución de los microdesechos en el área excavada del nivel 18b nos muestra que hay una mayor concentración de elementos en la K 14, L 14 y en los sectores colindantes de la K 15 y L 15 con las cuadrículas anteriores. Dentro de este área destacan tres picos de máxima densidad que coinciden con la zona limítrofe superior entre la L 14 y L 15, la zona limítrofe entre la K 14 y K 15 , y en el espacio de contacto entre K 13, K 14, L 13 y L 14. De modo más general, también se aprecia que el área comprendida entre la I 14 y la L 14 alberga una franja densa de microdesechos en este

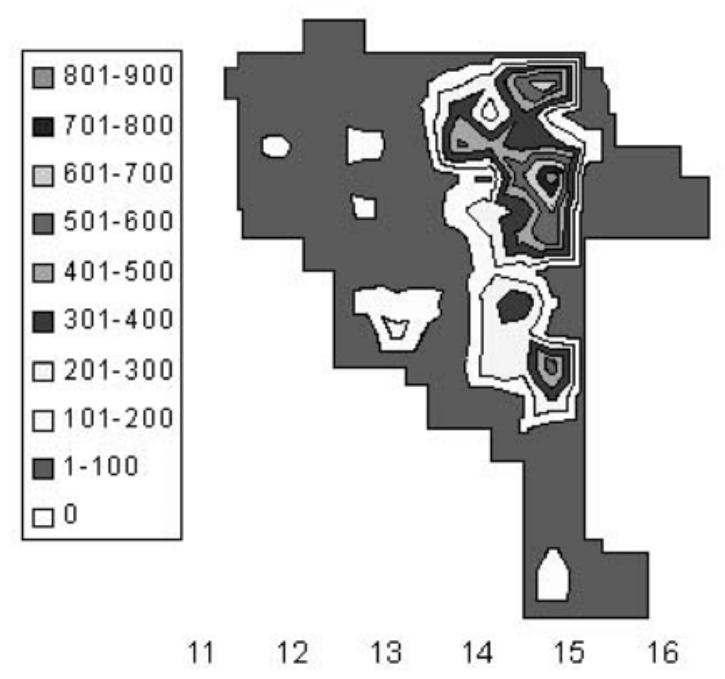

M

Fig. 5. Distribución de microdesechos en el nivel 18 B por cuadrícula. nivel. En el resto de la zona excavada, a pesar de la existencia de algún pequeño pico de densidad, se observa una distribución cuantitativa más o menos uniforme de los elementos.

\section{DISCUSIÓN}

A través de los resultados obtenidos se pueden realizar inferencias sobre el conjunto de microdesechos analizados. La identificación y análisis de las materias primas empleadas para la talla de los útiles encontrados en niveles arqueológicos nos puede proporcionar datos importantes para constatar las posibles estrategias tecnológicas desarrolladas por los grupos humanos. En el nivel 18b, hemos registrado una presencia predominante de la cuarcita 1 $(55,85 \%)$ frente al resto de materias primas presentes. En este sentido, Cabrera Valdés et al. (1996) ya observaron una presencia mayoritaria de cuarcita 1 en las capas coincidentes con el Auriñaciense Arcaico de El Castillo. La comparación (Tabla 14) entre las materias primas encontradas en los microdesechos del $18 \mathrm{~b}$ y las empleadas en los útiles ( $\mathrm{N}=240)$ de este mismo nivel nos permite observar:

1. En los microdesechos se registra arenisca mientras que en los útiles hay ausencia de esta materia prima.

2. Un bajo porcentaje de microdesechos en cuarcita $2(0,68 \%)$ en relación a su frecuencia en los útiles $(7,92 \%)$.

3. El porcentaje de microdesechos en cuarzo $(5,90 \%)$ y caliza $(22,11 \%)$ es elevado con respecto al de los útiles $(0,41 \%$ y $5,83 \%$ respectivamente) de estas materias primas.

4. Para el resto de materias primas la diferencia porcentual entre microdesechos y útiles no es muy significativa.

\begin{tabular}{|l|r|r|r|r|}
\hline & \multicolumn{2}{|c|}{ Microdesechos } & \multicolumn{2}{c|}{ Útiles } \\
\hline Materia prima & \multicolumn{1}{|c|}{$\mathbf{N}$} & \multicolumn{1}{c|}{$\%$} & \multicolumn{1}{c|}{$\mathbf{~}$} & \multicolumn{1}{c|}{} \\
\hline Arenisca & 70 & 0,45 & 0 & 0 \\
\hline Cuarcita 1 & 8.595 & 55,87 & 159 & 66,25 \\
\hline Cuarcita 2 & 104 & 0,58 & 19 & 7,92 \\
\hline Cuarcita 3 & 261 & 1,70 & 4 & 1,67 \\
\hline Cuarzo & 3.401 & 22,11 & 14 & 5,83 \\
\hline Indiferenciada & 908 & 5,90 & 1 & 0,41 \\
\hline Limonita & 89 & 0,58 & 0 & 0,00 \\
\hline Ofita & 183 & 1,19 & 2 & 0,83 \\
\hline Sílex & 1.748 & 11,36 & 37 & 15,42 \\
\hline Total general & $\mathbf{1 5 . 3 8 5}$ & $\mathbf{1 0 0 , 0 0}$ & $\mathbf{2 4 0}$ & $\mathbf{1 0 0 , 0 0}$ \\
\hline
\end{tabular}

Tab. 14. Relación porcentual entre útiles y microdesechos por materia prima. 
De acuerdo con estos resultados se podría inferir que la arenisca fue tallada en el yacimiento, si bien sus correspondientes útiles no fueron hallados en el área excavada. De este modo, se podría decir que los microdesechos de arenisca forman un "ghost" con respecto a sus útiles (Morrow 1996).

El bajo porcentaje de microdesechos $(13,31 \%)$ que albergan presencia de corteza (incluyendo a las lascas primarias y secundarias) podría deberse a que las materias primas hubieran entrado descortezadas o en un estado avanzado de reducción en la que las lascas grandes y núcleos pudieron conservar parte de la corteza en alguna de sus caras (Cowan 1999:604; Nash 1996:86). Es destacable el elevado porcentaje de cuarcita 1 con resto de corteza $(83,82 \%)$, esto puede responder a que pudo haber ingresado en un estado de menor desbastado que el resto de las materias primas. El sílex, por el contrario, presenta un índice de presencia de corteza muy bajo $(2,07 \%)$ en comparación con el porcentaje que representa respecto del total $(14,59 \%)$ de materias primas ( $\sin$ tener en cuenta las calizas), lo que podría indicar que el sílex registrado en el yacimiento ingresó en una fase avanzada de la cadena operativa.

En cuanto al estado de fragmentación, aunque se constata un ligero predominio de las lasca enteras, también se observa que las lascas indiferenciadas junto con las fracturadas con y sin talón dominan el conjunto. Esto coincide con lo propuesto por Ingbar et al. (1989:120-121) y Sullivan y Rozen (1985:762-763), según los cuales los desechos derivados de las actividades de talla de útiles alcanzan un porcentaje mayor de indiferenciados y fracturados. Por lo tanto, según estos autores, se habrían llevado a cabo en este nivel actividades involucradas en la formatización, regularización y mantenimiento de utensilios.

La fracturación de los microdesechos podría explicarse por el uso de percutores duros en el proceso de talla, a diferencia del uso de percutores blandos y la talla por presión que producen más cantidad de lascas enteras (Patterson y Sollberger 1978). Otras posibles causas podrían ser la acción del pisoteo humano (ver entre otros a Clark 1986; Maíllo Fernández 1998; Villa 1982), o de otros animales (Bocek 1986; Gómez 1996; Hull 1987; Gifford 1978; Peretti y Curtoni 1999; Villa 1982), y las propiedades particulares de talla de algunas materias primas como la cuarcita y el cuarzo. Éstas se fracturan fácilmente, al tiempo que desprenden un número elevado de astillas, esquirlas, y microdesechos
(Nami 1992 b). Desde nuestro punto de vista, el alto índice de fracturas y de lascas indiferenciadas detectado en la cuarcita 1 y el cuarzo, tiene relación con las condiciones particulares de las mismas.

Teniendo en cuenta la presencia de lascas angulares, planas y de reactivación directa se puede afirmar que se han desarrollado actividades relacionadas con la manufactura, mantenimiento y/o regularización de utensilios. La existencia de lascas de reactivación $(\mathrm{N}=347)$ es un indicador evidente de la prolongación de la vida útil de los utensilios (Bamforth 1986; Bellelli et al., 1985-1987; Binford 1979; Shott 1989). El análisis de las variables materia prima por tipo de lasca nos indica que las materias primas con un alto índice de lascas primarias y secundarias presentan un bajo porcentaje de lascas de reactivación y un número elevado de lascas indeferenciadas (por estado); de igual manera, las materias primas con una relativa alta frecuencia de lascas de reactivación directa presentan un bajo número de indiferenciadas y un alto de lascas enteras. De esta observación, se deduce que las materias primas que ingresaron al yacimiento en estado de menor desbastado (cuarzo, limonita y arenisca) apenas fueron sometidas actividades de mantenimiento y reavivado de los filos; por el contrario, las materias primas que entraron en etapas más avanzadas de la cadena operativa (sílex) estuvieron más sometidas a estas actividades. Este comportamiento podría estar en función de la mayor o menor cercanía de las fuentes de aprovisionamiento de estas materias o de las mejores o peores condiciones de las mismas para su talla y uso.

Los resultados derivados del análisis del módulo de longitud anchura no altera, en ningún modo, la constatación del desarrollo de actividades enfocadas a la manufactura, mantenimiento y/o regularización de útiles si tenemos presente la alta frecuencia de los módulos medios como subproductos de estas actividades. Es reseñable la escasa presencia de módulos laminares $(7,18 \%)$. Por su parte, los resultados de los espesores permiten apoyar las ideas planteadas en lo concerniente a las actividades de talla mencionadas anteriormente.

Las variables de talones y bulbos nos han facilitado información relevante a nivel tecnológico. La elevada proporción de talones preparados $(91,43 \%)$ (talones cuya superficie es parte del negativo de una o varias extracciones anteriores) en oposición a los no preparados o corticales $(6,85 \%)$ evidencia claramente que las actividades orientadas a las últimas etapas de reducción lítica se han desarrollado en 
mayor medida que las primeras etapas. Los talones preparados también son indicadores de estrategias conservadoras (Nash 1996:88). La combinación de resultados de los talones y bulbos en diversas materias primas (los tres tipos de cuarcita y el sílex) ha permitido observar algunas tendencias en las técnicas de talla: 1) la alta presencia de talones lisos y bulbos difusos es probablemente derivada de la aplicación de percutores blandos, y 2) de igual forma, los talones filiformes y puntiformes parecen ser característicos de la talla por presión (Espinosa 1995; Nami 1991 a y b; Patterson y Sollberger 1978). Algunos estudios ponen en relación la aplicación de percutores duros con los bulbos pronunciados (Baumler 1985; Bergman y Roberts 1988; Patterson y Sollberger 1978) en menor medida que los blandos.

El análisis por materia prima nos sugiere algunas particularidades. Por un lado, las cuarcitas tienen un comportamiento semejante: 1) se observa un alto número de talones lisos (sobre todo en cuarcita 2 y 3), 2) dominan los bulbos difusos seguidos de los indiferenciados, 3) es destacable el hecho de que los talones filiformes y puntiformes van aparejados con bulbos indiferenciados en mayor medida que con los pronunciados. Estos datos contrastan con los obtenidos para el sílex, en donde se registra para este tipo de talones (filiformes y puntiformes) una relación más próxima entre bulbos pronunciados e indiferenciados. En las cuarcitas también es reseñable el alto índice de talones corticales (en torno a un $8 \%$ ). Este dato apoyaría la idea ya antes comentada del ingreso al yacimiento de la cuarcita (en mayor medida que el resto de materias primas) en estados iniciales de reducción lítica. En cuanto al sílex, es interesante destacar: 1) el elevado porcentaje de talones filiformes y puntiformes; 2) la existencia de un mayor número de bulbos pronunciados para este tipo de talones, con respecto a las otras materias primas, que podría deberse a las particulares propiedades del sílex para la talla; y 3) la casi nula presencia de talones corticales, lo que nuevamente apoyaría la idea de que el sílex entraría al yacimiento en un estado avanzado de descortezado.

La presencia de lascas de reactivación $(\mathrm{N}=347)$ distribuidas en todas las cuadrículas del área excavada indica el desarrollo de actividades de reavivado y mantenimiento de los filos en los útiles (Binford 1979; Dibble 1984; Fish 1981; Kuhn 1991; Stevenson 1985; entre otros), principalmente sobre sílex (porcentualmente).

El estudio de los útiles del nivel $18 \mathrm{~b}$ permite pensar que se han desarrollado preferentemente estrategias tecnológicas conservadoras (curated) con respecto a las expeditivas (expedient) siempre y cuando se considere que las estrategias conservadoras producen conjuntos que son tecnológicamente sofisticados y probablemente distintos en su forma, donde los utensilios individuales serán destinados para una variedad de propósitos anticipados, mantenidos para un número indeterminado de usos, transportados entre yacimientos para estos usos y reciclados para otras tareas (Bamforth 1986:38; Binford 1979:269-270; Shott 1989:24). La industria lítica del nivel $18 b(\mathrm{~N}=240)$ se caracteriza, en primer lugar, por utensilios con un mayor grado de inversión de energía en su elaboración (Bamforth 1986:38). Esta compuesto por una elevada representatividad del grupo de raspadores (33 en cuarcita, 8 en sílex, 5 en calizas, 1 en ofita y 1 en limonita), seguidos por el grupo de piezas retocadas (raederas y piezas con retoque continuo; 60 en cuarcita, 10 en sílex y 2 en caliza), perforadores (11 en cuarcita, 2 en limonita, 1 en caliza y 1 en sílex), buriles (13 en cuarcita, 3 en sílex, 1 en caliza y 1 en cuarzo), puntas ( 1 en cuarcita) y, por último, el grupo de útiles compuestos (piezas retocadas que a su vez presentan una muesca, un raspador u otro útil; 8 en cuarcita) (Cabrera Valdés et al. 2001). En segundo lugar, se encuentra un conjunto de útiles que tienen un menor costo energético en su elaboración lo que implicaría estrategias tecnológicas expeditivas que producen conjuntos que son tecnológicamente más simples, la manufactura de utensilios sería una respuesta inmediata a tareas específicas y éstos serían descartados posteriormente a su uso (Binford 1979:269; Bamforth 1986:38). Estos útiles se encuentran comprendidos por el grupo de piezas escotadas (11 en cuarcita, 4 en sílex y 3 en caliza), seguidas por el grupo de piezas denticuladas ( 11 en cuarcita, 2 en sílex, 1 en caliza y 1 en limonita) y el grupo de piezas truncadas ( 5 en cuarcita). De la totalidad de utensilios $(\mathrm{N}=240)$, la materia prima más representada es la cuarcita $(75,84 \%)$, seguida por el sílex $(15,42 \%)$, la caliza $(5,83 \%)$ y la limonita $(1,67 \%)$; la ofita y la limonita no superan el $1 \%$. Como se puede observar, las materias primas usadas en la manufactura de los diferentes utensilios son preferentemente cuarcita y sílex y, en menor medida, caliza y limonita (Cabrera Valdés et al. 2001). Sin embargo, a pesar de que las estrategias conservadoras y expeditivas se han constatado en esta yacimiento, éstas no deberían considerarse como únicas y excluyentes según lo 
propuesto por Nelson, en donde el oportunismo (opportunistic) no esta planificado y responde a condiciones inmediatas y no anticipadas (Nelson 1991:62-66).

El bajo número y la dispersión de los microdesechos quemados encontrados no permite realizar con total seguridad ninguna inferencia. En nuestra opinión, podrían ser el resultado de las actividades de limpieza llevadas a cabo en los hogares y posteriormente transportados y depositados en estas áreas.

En la distribución espacial de estos microdesechos se localizan unos picos y áreas de elevada densidad que pueden ser el resultado de: 1) acumulaciones de carácter primario derivadas de las actividades de talla in situ (Gould 1981; MoholoyNagy 1990), 2) acumulaciones secundarias producto de actividades de limpieza, y 3 ) finalmente, también podrían deberse en parte a procesos naturales y postdepositacionales que pueden actuar y afectar a los niveles arqueológicos (Peretti y Mingo 2000). En este sentido, hay que destacar que el estrato correspondiente a este nivel se acuña hacia el exterior de la cueva, siendo por tanto más gruesa la potencia del estrato en la franja del interior coincidente con las cuadrículas de número 14 y el área excavada de las 15 que en la parte más exterior del área excavada. La existencia de sectores con poca o nula presencia de microdesechos (sobre todo en las áreas de alta densidad) se debe a la localización en estos puntos de bloques calizos de tamaño variable, y que por tanto no fueron excavados. Finalmente, dado que el nivel 18a y el nivel 17 son estériles, no cabría la posibilidad de pensar que entre el conjunto de microdesechos del nivel $18 \mathrm{~b}$ existiesen algunos con origen en los niveles anteriores. En nuestra opinión, consideramos que, debido al reducido tamaño y al elevado número de los microdesechos analizados, esta acumulación responde fundamentalmente a la talla en estas áreas.

\section{CONCLUSIONES}

Las conclusiones que se derivan de este trabajo deben considerarse preliminares, hasta que se puedan integrar los resultados aquí obtenidos con los provenientes del estudio de la totalidad de los microdesechos líticos recuperados en los demás niveles involucrados en la transición del Paleolítico Medio al Superior. Esta es la razón que impide formular conclusiones de orden más general relativas a las actividades realizadas en el sitio o a la dedicación funcional en cada una de las áreas.

En cualquier caso, el análisis de los microdesechos recogidos en el nivel $18 \mathrm{~b}$ de la cueva de $\mathrm{El}$ Castillo nos ha permitido obtener la siguiente información:

1. Presencia mayoritaria de la cuarcita $(\mathrm{C} 1)$ en relación con el resto de materias primas presentes en el registro.

2. Bajo índice de corteza, lo que estaría indicando que las primeras etapas de la cadena operativa se desarrollaron en otro área. La cuarcita (C1) ingresó en el yacimiento en un estado de menor desbastado que el resto de las materias primas.

3. De acuerdo con los resultados de talones y bultos se podría deducir una mayor aplicación de percutores blandos, aunque también se advierte la aplicación de percutores duros.

4. Constatación del desarrollo de actividades de elaboración, modificación y mantenimiento opcional de los útiles

5. Mantenimiento y reavivado preferencial de los utensilios elaborados en sílex.

6. Los resultados derivados de este análisis y el examen de los útiles líticos indican una preferencia de las estrategias tecnológicas conservadoras en detrimento de las expeditivas.

7. Existencia de una distribución espacial diferencial de los microdesechos.

8. Posible identificación de áreas de talla in situ (sin descartar aportes de carácter secundario).

Consecuentemente, afirmamos que el análisis de microdesechos líticos es ciertamente relevante, ya que, como hemos visto, puede orientar a identificar y detectar las actividades y procesos que derivan del subsistema de producción lítico, y puede facilitar un mejor y más preciso entendimiento de la dinámica de formación de los registros arqueológicos.

\section{BIBLIOGRAFÍA}

ASCHER, R. 1961: "Experimental Archaeology”. American Anthropologist 63:793-816.

ASCHERO, C. 1975: Ensayo para una Clasificación Morfológica de Artefactos Líticos Aplicada a Estudios Tipológicos Comparativos. Informe presentado al CONICET. Buenos Aires. MS.

- 1983: Ensayo para una Clasificación Morfológica de Artefactos. Apéndice A y B. Cátedra de Ergología y Tecnología. Facultad de Filosofía y Letras. Universidad de Buenos Aires. MS. 
BAGOLINI, B. 1968: Ricerche sulle Dimensioni dei Manufatti Litici Prehistorici non Ritocatti. Annali dell' Universita di Ferrara. Nuova Serie. Sezione XV. I (10):195-219.

BAMFORTH, D. 1986: “Technologycal Efficiency and Tool Curation". American Antiquity 51 (1): 38-50.

BAUMLER, M. 1985: "On the Interpretation of Chipping Debris Concentrations in the Archaeological Record". Lithic Technology 14 (3):120-125.

BAUMLER, M. y DOWNUM, C. 1989: "Between Micro and Macro: a Study in the Interpretation of Small-Size Lithic Debitage". En E. Amick (ed.): Experiments in Lithic Technology. B. A. R. International Series 528:101116.

BELLELLI, C. ; GURAIEB, G. y GARCÍA, J. 1985-1987: "Propuesta para el Análisis y Procesamiento por Computadora de Desechos de Talla Lítica (DELCO. Desechos Líticos Computarizados)". Arqueología Contemporánea 2(1):36-53.

BERGMAN, C y ROBERTS, M. 1988: "Flaking Techniques at the Acheulean Site of Boxgrove, West Sus-sex (England)". En A. Tuffreau (ed.): Culture $s$ et Industries Litiques en Milieu Loessique Revue Archéologique de Picardie 1-2: 105-112. Amiens, France.

BERNALDO DE QUIRÓS, F. y CABREVA VALDÉS, V. 1996: "Economical Strategies in the Upper Paleolithic in the Cantabrian Region". Human Evolution 11(2): 121-128.

BINFORD, L. 1978: "Dimensional Analysis of Behavior and Site Structure: Learning from Eskimo Hunting Stand". American Antiquity 43:330-361.

- 1979: "Organization and Formation Processes: Looking at Curated Technologies". Journal of Anthropological Research 35 (3):255-273.

BOCEK, B. 1986: "Rodent Ecology and Burrowing Behavior: Predicted Effects on Archaeological Site Formation". American Antiquity 51(3):589-603.

- 1992: "The Jasper Ridge Reexcavation Experiment: Rates of Artifact Mixing by Rodents". American Antiquity 57(2):261-269.

CABRERA VALDÉS, V. y BISCHOFF, J. 1989: “Accelerator Radiocarbon Dates for Early Upper Paleolithic (Basal Aurignacian) at El Castillo Cave (Spain)". Journal of Archaeological Science 16: 577-584.

CABRERA VALDÉS, V.; HOYOS, M. y BERNALDO DE QUIRÓS, F. 1993: "La Transición del Paleolítico Medio / Paleolítico Superior en la Cueva de El Castillo: Características Paleoclimáticas y Situación Cronológica". En V. Cabrera (ed.): El Origen del Hombre Moderno en el Suroeste de Europa. Universidad Nacional de Educación a Distancia. Madrid: 81-101.

CABRERA VALDÉS, V.; LLORET, M. y BERNALDO DE QUIRÓS, F. 1996: "Materias Primas y Formas Líticas del Auriñaciense Arcaico de la Cueva del Castillo (Puente Viesgo, Cantabria)". Espacio, Tiempo y Forma. Serie 1, Prehistoria y Arqueología 9: 141158.

CABRERA VALDÉS, V. y BERNALDO DE QUIRÓS, F. 1996: "El Hombre Moderno en Cantabria: La Transición del Paleolítico Medio al Paleolítico Superior a través de la Revisión Estratigráfica de la Cueva del Castillo". Actas del Primer Encuentro de Historia de Cantabria. Universidad de Cantabria y Gobierno de Cantabria. Santander 1: 129-147.

CABRERA VALDÉS, V.; LLORET, M.; BERNALDO DE QUIRÓS, F. y HOYOS, M. 1997: "El Auriñaciense Arcaico de la Cueva de El Castillo”. En R. De Balbín y P. Bueno (eds.): Actas del II Congreso de Arqueología Peninsular. I: Paleolítico y Epipaleolítico. Fundación Rei Afonso Henriques: 133-140.

CABRERA VALDÉS, V.; MAÍLLO, J.M. ; LLORET, M. y BERNALDO DE QUIRÓS, F. 2001: "La Transition vers le Paléolithique Supérieur dans la Grotte du Castillo (Cantabrie, Espagne): La Couche 18. L'Anthropologie 105: 505-532.

CAHEN, D Y J. MOEYERSONS 1977: "Subsurface Movements of Stone Artifact and Their Implications for the Prehistory of Central Africa”. Nature 266: 812-815.

CLARK, J. 1986: "Another Look at Small Debitage and Microdebitage". Lithic Technology 15: 21-33.

COWAN, F. 1999: "Making Sense of Flake Scatters: Lithic Technological Strategies and Mobility". American Antiquity 64 (4): 593-607.

DIBBLE, H. 1984: "Interpreting Typological Variations of Middle Paleolithic Scrapers: Functions, Style or Sequence of Reduction?". Journal of Field Archaeology 11: 431-436.

DUNNELL, R. y STEIN, J. 1989 : "Theoretical Issues in the Interpretation of Micro-artifacts". Geoarchaeology 4:31-42.

ESPINOSA, S. 1995: "Dr. School y Monsier Fleur: de Talones y Bulbos". Cuadernos 16: 315-328.

FISH, P. 1981: "Beyond Tools: Middle Palaeolithic Debitage Analysis and Cultural Inference". Journal of Anthropological Research 37: 374386.

FLADMARK, K. 1982: "Microdebitage Analysis: Initial Considerations". Journal of Archaeological Science 9:205-220.

GALLAGHER, J. 1977: "Contemporary Stone Tools in Ethiopia: Implications for Archaeology". Journal of Field Archaeology 4: 407-414.

GIFFORD, D. 1978: "Ethnoarchaeological Observations on Natural Processes Affecting Cultural Materials". En R. Gould (ed.): Explorations in Etnoarchaeology: 77-102. University of New Mexico Press. Albuquerque.

GÓMEZ, G. 1996: Los Pequeños Mamíferos del Sitio Arroyo Seco 2 (Ptdo. de Tres Arroyos, Pcia. de Bs. As.). Aspectos Relacionados con la Subsistencia, Tafonomía y el Paleoclima. Tesis de Licenciatura. Facultad de Ciencias Sociales. Universidad Nacional del Centro. Olavarría. MS. 
GOULD, R. 1979: "Exotic Stones and Battered Bones. Ethnoarchaeology in the Australian Desert". Archaeology 32: 229-237.

- 1981: "Brandon Revisted: A New Look at an Old Technology”. En R. Gould y M. B. Schiffer (eds.): Modern Material Culture: The Archaeology of Us: 269-281. Academic Press. New York.

HASSAN, F. 1978: "Sediments in Archaeology: Methods an Implications for Paleoenvironmental and Cultural Analysis". Journal of Field Archaeology 5:197-213.

HEDGES, R.; HOUSTLEY, R.A.; RAMSEY, C.B. y VAN KLINKEN, G.J. 1994: "Radiocarbon Dates from the Oxford AMS System: Archaeometry Datelist 18". Archaeometry 36 (2): 337-374.

HOYOS, M. 1979: El Karst en Asturias en el Pleistoceno Superior y Holoceno. Tesis Doctoral. Universidad Complutense. Madrid.

HULL, K. 1987: "Identification of Cultural Site Formation Processes Through Microdebitage Analysis". American Antiquity 54: 851-855.

INGBAR, E.; LARSON, M. y BRADLEY, B. 1989: “A None Tipologycal Approach to Debitage Analysis". In D. Amick y Maulding (eds.): Experiments in Lithic Technology. British Archaeologycal Reports International Series 528: 17-135. Oxford.

KUHN, S. 1991: "Unpacking Reduction: Lithic Raw Material Economy in the Mousterian of West-Central Italy". Journal of Anthropological Archaeology 10:76-106.

McBREARTY, S.; BISHOP, L.; PLUMMER, T.; DEWARD, R. y CONARD, N. 1998: "Tools Underfoot: Human Trampling as an Agent of Lithic Artifact Edge Modifications". American Antiquity 63(1):108-129.

MAÍLLO FERNÁNDEZ, J. 1998: "Proporciones de Debris en Réplicas de Talla Experimental". Espacio, Tiempo y Forma, Serie 1, Prehistoria y Arqueología 11:45-55.

MOHOLOY-NAGY, M. 1990: "The Misidentification of Mesoamerican Lithic Workshop". Latin American Antiquity 1:268-279.

MORROW, T. 1996: "Lithic Refitting and Archaeological Site Formation Processes. A case Study from the Twin Ditch Site, Greene County, Illinois". En G. Odell (ed.): Stone Tools Theoretical Insights into Human Prehistory. 345-373. Plenum Press. New York y London.

NAMI, H. 1991a: "Desechos de Talla y Teoría de Alcance Medio: un Caso de Península Mitre, Tierra del Fuego". Actas X Congreso Nacional de Arqueología Argentina. San Fernando del Valle de Catamarca. Shincal 3 (2): 94-112.

- 1991b: "Algunas Reflexiones Teóricas sobre Arqueología y Experimentación”. Actas X Congreso Nacional de Arqueología Argentina. San Fernando del Valle de Catamarca. Shincal 3 (1):151-168.

- 1992a: "Informe sobre el Primer Curso de Análisis de Desechos de Talla Experimentales en Argentina". $\mathrm{Pa}$ limpsesto. Revista de Arqueología 1:75-79.

- 1992b: "El Subsistema Tecnológico de la Confección de Instrumentos Líticos y la Explotación de los Recursos del Ambiente: una Nueva Vía de Aproximación". Shincal 2: 33-53.

NAMI, H. y BELLELI, C. 1994: "Hojas, Experimentos y Análisis de Desechos de Talla. Implicaciones Arqueológicas para la Patagonia Centro-Septentrional”. Сuadernos del Instituto Nacional de Antropología 15:199223.

NASH, S. 1996: "Is Curation Useful Heuristic?". En G. Odell (ed.): Stone Tools Theoretical Insights into $\mathrm{Hu}$ man Prehistory 81-99. Plenum Press. New York y London.

NELSON, M. 1991: "The Study of Technological Organization”. En M. B. Schiffer (ed.): Archaeological Method and Theory 3: 57-100. Tucson, Arizona.

PATTERSON, L. y SOLLBERGER, P. 1978: "Replication and Classification of Small Size Lithic Debitage". Plains Anthropologist 23 (80): 103-112.

PERETTI, R. 1997: Estudio de Microdesechos Líticos en el Sitio Arqueológico Arroyo Seco 2 (Pdo. De Tres Arroyos. Pcia. De Buenos Aires): Nuevas Vías de Aproximación a la Comprensión del Subsistema Tecnológico Lítico. Tesis de Licenciatura. Facultad de Ciencias Sociales. Universidad Nacional del Centro. Olavarría. MS.

PERETTI, R. y CURTONI, R. 1999: Entraron enteras y salieron rotas: Lascas experimentales y procesos deformación en conjuntos líticos. MS.

PERETTI, R. y MINGO, A. 2000: "Estudios Tafonómicos del Nivel Auriñaciense Arcaico de la Cueva de El Castillo (Puente Viesgo, Cantabria): Los Microdesechos Líticos”. Espacio, Tiempo y Forma, Serie I, Prehistoria y Arqueología 13: 89-124.

SCHIFFER, M. B. 1972: "Archaeological Context and Systemic Context”. American Antiquity 37 (2):156165.

- 1983: "Toward the Identification of Formation Processes". American Antiquity 48 (4): 675-706.

- 1987: Formation Processes of the Archaeological Record. University Of New Mexico Press, Albuquerque.

SHOTT, M. 1989: "Diversity, Organization, and Behavior in the Material Record: Ethnografic and Archaeological Examples". Current Anthropology 30 (3): 283-315.

- 1994: "Size and Form in the Analysis of Flake Debris: Review and Recent Approaches". Journal of Archaeological Method and Theory 1 (1): 69-110.

STEVENSON, M. 1985: "The Formation of Artifact Assemblages at Workshop/Habitation sites:models from Peace Point in Northern Alberta". American Antiquity 50 (1): 63-81.

SULLIVAN, A. y ROZEN, K. 1985: "Debitage Analysis and Archaeological Interpretation". American Antiquity 50 (4): 755-759.

VILLA, P. 1982: "Conjoinable Pieces and Site Formation Processes”. American Antiquity 47: 276-290. 